QUARTERLY OF APPLIED MATHEMATICS

VOLUME LXVII, NUMBER 1

MARCH 2009, PAGES 125-136

S 0033-569X(09)01086-4

Article electronically published on January 7, 2009

\title{
THERMAL GROOVING BY SURFACE DIFFUSION: MULLINS REVISITED AND EXTENDED TO MULTIPLE GROOVES
}

\author{
BY \\ P. A. MARTIN \\ Department of Mathematical and Computer Sciences, Colorado School of Mines, Golden, Colorado \\ 80401-1887
}

\begin{abstract}
We extend the original Mullins theory of surface grooving due to a single interface to multiple interacting grooves formed due to closely spaced flat interfaces. First, we show that Mullins' analysis for one groove can be simplified by using Fourier cosine transforms instead of Laplace transforms. Second, we solve the corresponding problem for an infinite periodic row of grooves. For both of these problems, symmetry considerations ensure that the interface conditions reduce to boundary conditions. Third, we solve the problem for two interacting grooves. Continuity requirements at the groove roots require sliding at the interfaces or tilting of the groove roots. We adopt the latter model. We find that the groove roots tilt until the surface curvature of the semi-infinite profiles is eliminated.
\end{abstract}

1. Introduction. It has been fifty years since the well-known paper by Mullins [6], entitled Theory of Thermal Grooving, was published. He considered the following initialvalue problem. A vertical flat grain boundary meets a horizontal flat surface. Immediately, the grain boundary forms a groove in the surface, with a known angle at the triple point. The groove becomes deeper with time, and the problem is to calculate the evolution of the free surface. For background and literature reviews, see [2], 3] and [4].

In fact, Mullins [6] gave two separate theories, one based on evaporation-condensation kinetics and one based on surface diffusion. In this paper, we focus on the latter mechanism; a similar and simpler analysis can be developed for evaporation-condensation kinetics.

Suppose that (in two dimensions) the surface profile is defined by a function $y(x, t)$, where $x$ is the horizontal coordinate and $t$ is time. Mullins [6] showed that the normal velocity of the surface, $v_{n}$, is proportional to the Laplacian of the mean curvature, $\kappa$. In two dimensions, this becomes

$$
v_{n}=B \partial^{2} \kappa / \partial \tilde{s}^{2},
$$

Received July 25, 2007.

2000 Mathematics Subject Classification. Primary 35R35.

E-mail address: pamartin@mines.edu

(C)2009 Brown University Reverts to public domain 28 years from publication 
where $\tilde{s}$ is the arclength along the surface. The parameter $B=D_{s} \gamma_{s} \omega /\left(k_{B} T\right)$ is a constant: $D_{s}$ is the surface diffusivity, $\gamma_{s}$ is the surface energy, $\omega$ is the atomic volume, $k_{B}$ is the Boltzmann constant, and $T$ is the absolute temperature. Equation (1) can be written as a nonlinear, fourth-order partial differential equation for $y(x, t)$,

$$
\frac{\partial y}{\partial t}=-B \frac{\partial}{\partial x}\left\{X \frac{\partial}{\partial x}\left(X^{3} \frac{\partial^{2} y}{\partial x^{2}}\right)\right\},
$$

with $X(x, t)=\left\{1+(\partial y / \partial x)^{2}\right\}^{-1 / 2}$.

In most crystalline systems, $\gamma_{s}$ is much larger than typical interfacial energies $\gamma_{i}$. Then, the small-slope approximation, $\kappa \simeq \partial^{2} y / \partial x^{2}(X \simeq 1)$, may be used, giving a linear equation for the evolution of the surface profile $y(x, t)[\underline{6}$,

$$
\frac{\partial y}{\partial t}+B \frac{\partial^{4} y}{\partial x^{4}}=0 .
$$

Mullins [6] solved (3) for a single groove, located at $x=0$. By symmetry, a problem posed in $x>0$ can be formulated. Mullins solved this problem using Laplace transforms with respect to $t$. This is a powerful method, and a natural choice for initial-value problems. However, we shall show in Sec. 2 that it is much simpler to use a Fourier cosine transform with respect to $x$.

Mullins' solution has been used extensively to measure interfacial energies and surface diffusivities. His solution has been generalized in various ways. One way is to incorporate nonlinear effects, using (2). This was done by Robertson [8] and then by Broadbridge and Tritscher 1, 9]. These papers consider the evolution of a single groove.

Here, we retain Mullins' linear model, (3), but we consider systems involving more than one groove. We do this because we expect that interactions between closely spaced grooves could result in surface profiles that differ significantly from those predicted by Mullins' one-groove solution. Specifically, if we have two grooves, distance $2 \ell$ apart, we ask: how do the grooves interact, how does the interaction depend on $\ell$, and how far apart do the grooves have to be before Mullins' one-groove solution gives a good approximation? We treat this problem in Sec. 4. In Sec. 3, we consider a simpler problem, in which the inherent asymmetry of the two-groove problem is eliminated. Thus, we solve the problem for an infinite, $2 \ell$-periodic row of grooves.

For the two-groove problem, suppose the grooves are at $x= \pm \ell$; evidently, the surface profile $y(x, t)$ is an even function of $x$, so that we can focus on the interface at $x=\ell$.

Introduce plane polar coordinates $(\rho, \varphi)$ so that $\rho=0$ is the groove root and the two sides of the groove approach the root along $\varphi= \pm \varphi_{\mathrm{g}}$, say. Thermodynamic equilibrium requires that the groove angle, $2 \varphi_{\mathrm{g}}$, be fixed. However, the line bisecting the groove (given by $\varphi=0$ ) need not be vertical: the groove-root system can tilt, or rotate, as $t$ increases. This tilting must be calculated as part of the solution. (A similar idea can by found in [7, where the authors consider a single groove in a step-like surface.) To solve this problem, we revert to Laplace transforms because we want to use the same method between the grooves $(|x|<\ell)$ and beyond the grooves $(|x|>\ell)$. The problem is solved exactly and then various properties of the solution are extracted. 
2. Mullins revisited: one groove. Mullins determined the symmetric semi-infinite groove profile due to a single interface at $x=0$ by solving (3) in the quadrant $x>0$, $t>0$, subject to

$$
\begin{aligned}
\partial y / \partial x & =\theta_{\mathrm{eq}} & & \text { at } x=0, t>0, \\
\partial^{3} y / \partial x^{3} & =0 & & \text { at } x=0, t>0, \quad \text { and } \\
y & =0 & & \text { at } t=0, x>0 .
\end{aligned}
$$

The condition (4) imposes the energetic balance of the dihedral angles at the triple junctions; $\theta_{\text {eq }}$, a positive constant, is the equilibrium dihedral angle at the surface-interface junction given by

$$
2 \theta_{\mathrm{eq}}=\gamma_{i} / \gamma_{s}
$$

Thus, the groove profile at the root has a fixed slope for all time. Condition (5) ensures that the contribution of grain boundary diffusion to the overall evolution is negligible compared to surface diffusion. The initial condition (6) says that the surface evolves from a flat surface. An immediate consequence of (3), (5), and (6) is mass conservation;

$$
\int_{0}^{\infty} y(x, t) d x=0 \quad \text { for all } t \geq 0 .
$$

Mullins [6] solved the problem for $y(x, t)$ using a Laplace transform with respect to $t$ (see Sec. 4.1 below). We give a simpler treatment, using a Fourier cosine transform with respect to $x$. Thus, define

$$
Y_{\mathrm{c}}(k, t)=\int_{0}^{\infty} y(x, t) \cos k x d x .
$$

Transforming (3) yields

$$
\partial Y_{\mathrm{c}} / \partial t+B k^{4} Y_{\mathrm{c}}=-B k^{2} \theta_{\mathrm{eq}},
$$

where we have used (4) and (5). Transforming the initial condition (6) gives $Y_{\mathrm{c}}(k, 0)=0$, and then we can easily solve (9):

$$
Y_{\mathrm{c}}(k, t)=-\theta_{\mathrm{eq}} k^{-2}\left(1-e^{-k^{4} B t}\right) .
$$

(Note that $Y_{\mathrm{c}}(0, t)=0$ in accordance with (8).) Inverting (10) gives

$$
y(x, t)=-\frac{2 \theta_{\mathrm{eq}}}{\pi} \int_{0}^{\infty}\left(1-e^{-k^{4} B t}\right) \frac{\cos k x}{k^{2}} d k .
$$

This is an explicit formula for the solution of Mullins' problem. Also notice that the problem has a single length scale, $(B t)^{1 / 4}$.

In particular, we can evaluate the solution at $x=0$ :

$$
\begin{aligned}
y(0, t) & =-\frac{2 \theta_{\mathrm{eq}}}{\pi} \int_{0}^{\infty}\left(1-e^{-k^{4} B t}\right) \frac{d k}{k^{2}} \\
& =-\frac{8 \theta_{\mathrm{eq}} B t}{\pi} \int_{0}^{\infty} k^{2} e^{-k^{4} B t} d k \\
& =-2\left(\theta_{\mathrm{eq}} / \pi\right)(B t)^{1 / 4} \Gamma\left(\frac{3}{4}\right) ;
\end{aligned}
$$


the second formula comes after an integration by parts and the last formula comes from the substitution $k^{4} B t=k^{\prime}$, say, and the definition of the gamma function,

$$
\Gamma(z)=\int_{0}^{\infty} x^{z-1} e^{-x} d x \text { for } \operatorname{Re}(z)>0 .
$$

The expression (12) agrees with [6, Equation (30)] when one notes that $\Gamma\left(\frac{3}{4}\right) \Gamma\left(\frac{5}{4}\right)=$ $2^{-3 / 2} \pi$.

Returning to (11), the substitutions $k=w /(B t)^{1 / 4}$ and $u=x /(B t)^{1 / 4}$ give

$$
y(x, t)=\theta_{\mathrm{eq}}(B t)^{1 / 4} Z(u),
$$

where Mullins' function $Z$ is given explicitly by

$$
\begin{aligned}
Z(u)= & -\frac{2}{\pi} \int_{0}^{\infty}\left(1-e^{-w^{4}}\right) \cos (u w) \frac{d w}{w^{2}} \\
= & Z(0)+\frac{2}{\pi} \int_{0}^{\infty}\{1-\cos (u w)\} \frac{d w}{w^{2}} \\
& +\frac{2}{\pi} \int_{0}^{\infty} \frac{\cos (u w)-1}{w^{2}} e^{-w^{4}} d w
\end{aligned}
$$

and $Z(0)=-(2 / \pi) \Gamma\left(\frac{3}{4}\right)$. An integration by parts shows that the second term on the right-hand side of (16) is equal to $u$. For the third term, we can insert the Maclaurin series for $\cos (u w)$ and then integrate term by term, using (13). The final result is

$$
Z(u)=\sum_{n=0}^{\infty} a_{n} u^{n}
$$

where $a_{0}=Z(0), a_{1}=1, a_{2 m+1}=0$ for $m=1,2,3, \ldots$, and

$$
a_{2 m}=\frac{1}{2 \pi} \frac{(-1)^{m}}{(2 m) !} \Gamma\left(\frac{2 m-1}{4}\right), \quad m=0,1,2, \ldots
$$

The ratio test shows that the series (17) is absolutely convergent for all $u$. Also, (8) gives $\int_{0}^{\infty} Z(u) d u=0$.

Mullins [6] obtained the first 15 coefficients in (17) by substituting (14) and (17) in (3), as in the method of Frobenius. Our solution shows that (17) converges rapidly for moderate values of $u$. Thus, the physics of surface evolution is captured by the first few terms of the power series (17). For large $u$ (that is, during the early stages of evolution away from the groove), we can integrate (15) by parts; this shows that $Z(u)$ decays faster than $u^{-n}$ for every positive integer $n$ : $Z(u)$ decays exponentially as $u \rightarrow \infty$.

We note that our method for solving the Mullins problem generalizes to physical situations where the energetic equilibrium at the groove root is time dependent. Examples include changes in interfacial (including surface) energy, interface structure transitions, and asymmetrical kinetics in the two phases abutting the interface. In general, suppose that the angle at the groove root, $\theta_{\mathrm{eq}}$, is replaced by a prescribed function of $t, \theta(t)$. Then, (9) would have $\theta(t)$ instead of $\theta_{\text {eq }}$ on the right-hand side, giving

$$
Y_{\mathrm{c}}(k, t)=-B k^{2} e^{-k^{4} B t} \int_{0}^{t} \theta(\tau) e^{k^{4} B \tau} d \tau .
$$


Inverting the cosine transform and interchanging the order of integration then gives

$$
y(x, t)=-\frac{2 B}{\pi} \int_{0}^{t} \theta(\tau) \int_{0}^{\infty} k^{2} \cos k x e^{-k^{4} B(t-\tau)} d k d \tau .
$$

The formulas (11) and (18) can also be obtained using Laplace transforms, but the calculations are much more complicated; see Sec. 4.1

Evaluating (18) at $x=0$, using (13) again, gives

$$
y(0, t)=-(2 \pi)^{-1} \Gamma\left(\frac{3}{4}\right) B^{1 / 4} \int_{0}^{t} \theta(\tau)(t-\tau)^{-3 / 4} d \tau .
$$

If $\theta$ has a Maclaurin expansion, $\theta(t)=\sum_{n=0} \theta^{(n)}(0) t^{n} / n$ !, the integral can be evaluated to give

$$
y(0, t)=-(B t / 4)^{1 / 4} \sum_{n=0} \frac{\theta^{(n)}(0) t^{n}}{\Gamma\left(n+\frac{5}{4}\right)}
$$

this reduces to (12) when $\theta(t) \equiv \theta_{\text {eq }}=\theta(0)$.

3. Multi-groove systems: periodic surface profile. In this section, we consider an infinite, $2 \ell$-periodic row of grooves. For this problem, we can exploit symmetry across each interface and then solve for $y$ within each period.

It is convenient to move the origin so that it is halfway between two adjacent grooves. Then, we consider the following problem for $y(x, t)$ : solve (3) for $-\ell<x<\ell$ and $t>0$, subject to

$$
\begin{aligned}
\partial y / \partial x & =\theta_{\mathrm{eq}} & & \text { at } x \\
\partial y / \partial x & =-\theta_{\mathrm{eq}} & & \text { at } x=\ell, t>0, \\
\partial^{3} y / \partial x^{3} & =0 & & \text { at } x=-\ell \text { and at } x=\ell, t>0, \text { and } \\
y & =0 & & \text { at } t=0,-\ell<x<\ell .
\end{aligned}
$$

Evidently, the solution of this problem is an even function of $x, y(-x, t)=y(x, t)$.

The finite range for $x$ suggests the method of separation of variables. To make this method work, we require homogeneous boundary conditions at $x= \pm \ell$. This is achieved by writing

$$
y(x, t)=y_{0}(x)+v(x, t)
$$

where

$$
y_{0}(x)=\frac{1}{2} \theta_{\mathrm{eq}}\left(\ell^{2}-x^{2}\right) / \ell ;
$$

this function satisfies (3) and (20)-(22), so that $v$ must satisfy (3) together with

$$
\begin{aligned}
\partial v / \partial x & =0 & & \text { at } x=-\ell \text { and at } x=\ell, t>0, \\
\partial^{3} v / \partial x^{3} & =0 & & \text { at } x=-\ell \text { and at } x=\ell, t>0, \quad \text { and } \\
v & =-y_{0} & & \text { at } t=0,-\ell<x<\ell .
\end{aligned}
$$

The problem for $v$ is readily solved, giving

$$
v(x, t)=\theta_{\mathrm{eq}} \ell \sum_{n=0}^{\infty} b_{n} e^{-B t \lambda_{n}^{4}} \cos \lambda_{n} x
$$


where $\lambda_{n}=n \pi / \ell$ and the coefficients $b_{n}$ are found by imposing the initial condition (26); we find $b_{0}=-\frac{1}{3}$ and $b_{n}=2(-1)^{n} /(n \pi)^{2}$ for $n=1,2, \ldots$. Hence, our final expression for the surface profile, valid for $-\ell<x<\ell$ and $t>0$, is

$$
y(x, t)=\frac{\theta_{\mathrm{eq}} \ell}{6}-\frac{\theta_{\mathrm{eq}} x^{2}}{2 \ell}+\frac{2 \theta_{\mathrm{eq}} \ell}{\pi^{2}} \sum_{n=1}^{\infty} \frac{(-1)^{n}}{n^{2}} e^{-\xi n^{4}} \cos \frac{n \pi x}{\ell},
$$

where $\xi=B t(\pi / \ell)^{4}$ is a dimensionless quantity.

The competition between the two length scales, $\ell$ and $(B t)^{1 / 4}$, leads to the following observations. As $t \rightarrow \infty, \ell$ is the sole length scale in the solution and (unlike the selfsimilar profile for the single groove) we have an explicit formula for the finite- $\ell$ surface profile,

$$
\lim _{t \rightarrow \infty} y(x, t)=\frac{1}{6} \theta_{\mathrm{eq}}\left(\ell-3 x^{2} / \ell\right) \equiv y_{\infty}(x),
$$

say. The limiting profile, $y_{\infty}(x)$, is a simple quadratic, with extrema proportional to $\ell$ : maxima evolve to a height of $\theta_{\text {eq }} \ell / 6$ above the initial flat surface $(y=0)$, while the minima evolve to a depth $\theta_{\text {eq }} \ell / 3$ below $y=0$. Increasing $\ell$ increases the extent of the extrema, until $\ell \approx(B t)^{1 / 4}$. The evolution is then given by Mullins' solution. Thus, the spacing between the interfaces may be seen as a tool that could be used to control the height and depth of the modified surface profile. The ratio of the depth of the groove to the distance between the maxima is independent of $\ell$ and equal to $4 / \theta_{\text {eq }}$, compared to $4.73 / \theta_{\text {eq }}$ for the single groove [6, Equation (19)]. Finally, notice that $\int_{-\ell}^{\ell} y_{\infty}(x) d x=0$, as expected from mass conservation.

As a special case of (27), we can determine the evolution of the surface at the groove $\operatorname{roots}(x= \pm \ell)$ :

$$
y( \pm \ell, t)=-\frac{\theta_{\mathrm{eq}} \ell}{3}+\frac{2 \theta_{\mathrm{eq}} \ell}{\pi^{2}} \sum_{n=1}^{\infty} \frac{1}{n^{2}} e^{-\xi n^{4}} .
$$

For large $\ell$, we expect to recover Mullins' solution, given by (12). In this limit, $\xi \rightarrow 0$, and so the series in (29) tends to $\sum_{n=1}^{\infty} n^{-2}=\pi^{2} / 6$, so that the leading contribution from the series precisely cancels the term $-\theta_{\text {eq }} \ell / 3$ on the right-hand side of (29). Thus, to take the limit $\ell \rightarrow \infty$, we need more information about the behaviour of the series in (29). This is provided by the following theorem.

Theorem 1. Define a function $f(\xi)$ for $\xi>0$ by

$$
f(\xi)=\sum_{n=1}^{\infty} \frac{1}{n^{2}} e^{-\xi n^{4}}
$$

Then

$$
f(\xi) \sim \frac{1}{6} \pi^{2}-\Gamma\left(\frac{3}{4}\right) \xi^{1 / 4}+E(\xi) \quad \text { as } \xi \rightarrow 0,
$$

where the error $E(\xi)$ is exponentially small.

This theorem is proved in an appendix, using Mellin transforms [5]. When it is applied to (29), we obtain exactly the Mullins solution (12) in the limit $\ell \rightarrow \infty$; moreover, we see that the difference between the two solutions is exponentially small, meaning that the Mullins solution gives a remarkably good approximation to the finite- $\ell$ solution at the interface. 
Note that we have not determined the exact form (or even the sign) of $E(\xi)$ for all $\xi$. However, it is easy to see that $E(\xi)>0$ for $\xi>3.25$. Defining $E(\xi)$ by equality in (31), we obtain $E(\xi)>\Gamma(3 / 4) \xi^{1 / 4}-\pi^{2} / 6$, as $f(\xi)>0$; setting the right-hand side to zero gives $\xi>3.25$.

In order to determine the minimum interfacial spacing $\ell$ above which we can safely employ Mullins' solution, we use well-known material parameters. Using values for Ag near its melting point, $B=10^{-26} \mathrm{~m}^{4} \mathrm{sec}^{-1}$ [6], and the fact that the leading term in the error $E(\xi)$ decays exponentially with increasing $\ell$ such that $\xi \leq 0.1$ is a reasonable approximation for application of Mullins' solution, we obtain $\ell \geq 10 t^{1 / 4} \mathrm{~nm}$. Since $99 \%$ of the evolution at the groove takes place in a matter of hours [6], the single groove solution can be safely used for multilayer surface profiles with layer thickness greater than $100 \mathrm{~nm}$.

4. Multi-groove systems: two grooves. In this section, we consider a symmetric bi-groove configuration with two identical interfaces. The groove system consists of a layer $a$ of thickness $2 \ell$ sandwiched between two semi-infinite layers $b$. Each groove has a known angle (dictated by thermodynamics at the triple point) but the line bisecting each groove need not be vertical: were we to assume this, we would find sliding discontinuities along the interfaces. Thus, we allow each groove-root system to tilt, and we have to calculate the amount of tilt (as a function of time) as part of the problem's solution.

In order to solve the resulting mathematical problems, it is convenient to use a common technique in the region between the two grooves and in the two semi-infinite regions on either side: we use Laplace transforms, as used originally by Mullins [6]. First, we solve a generalization of the Mullins problem (Sec. 4.1) and a generalization of the periodicgroove problem (Sec. 4.2) in which the angle $\theta_{\text {eq }}$ is replaced by angles that can vary with time in a prescribed manner. These two solutions are then patched together properly, so as to solve the two-groove problem (Sec. 4.3).

To begin, we define the Laplace transform of $y$ with respect to $t$,

$$
Y(x, s)=\int_{0}^{\infty} y(x, t) e^{-s t} d t .
$$

Transforming (3) gives

$$
\partial^{4} Y / \partial x^{4}+(s / B) Y=0
$$

having used the initial condition $y(x, 0)=0$. We now solve (32), subject to various conditions.

4.1. A generalized Mullins problem. Suppose that we generalize the Mullins problem so that the slope at $x=0$ varies with time. Thus, we replace (4) with

$$
\partial y / \partial x=\theta(t) \quad \text { at } x=0, t>0
$$

where $\theta(t)$ is a given function with Laplace transform $\Theta(s)$. Then, we want to solve (32) for $x>0$, subject to

$$
\partial Y / \partial x=\Theta(s) \quad \text { and } \quad \partial^{3} Y / \partial x^{3}=0 \quad \text { at } x=0,
$$


with $Y \rightarrow 0$ as $x \rightarrow \infty$. The substitution $Y=e^{\lambda x}$ in (32) leads to

$$
\lambda^{4}+(s / B)=0
$$

an equation for $\lambda$. In general, the transform variable $s$ could be complex, but, for now, it is sufficient to suppose that $s$ is real and positive. Writing $\lambda=\Lambda e^{i \varphi}$, we obtain

$$
\Lambda=(s / B)^{1 / 4} \text { and } \varphi=(2 n+1) \pi / 4, \quad n=0, \pm 1, \pm 2, \ldots .
$$

We pick two values of $\varphi, \varphi_{1}$ and $\varphi_{2}$, that give two different values of $\lambda, \lambda_{1}$ and $\lambda_{2}$, respectively, with $\operatorname{Re}(\lambda)<0$ (to ensure decay as $x \rightarrow \infty$ ). We take $\varphi_{1}=3 \pi / 4$ and $\varphi_{2}=-3 \pi / 4$, giving $\lambda_{1}=(i-1) \Lambda / \sqrt{2}$ and $\lambda_{2}=\overline{\lambda_{1}}$, the complex conjugate of $\lambda_{1}$. Thus, we have

$$
Y(x, s)=\mathcal{A}(s) e^{\lambda_{1} x}+\mathcal{B}(s) e^{\lambda_{2} x},
$$

where $\mathcal{A}$ and $\mathcal{B}$ are determined using the boundary conditions (33), giving

$$
Y(x, s)=\frac{\Theta(s)}{\lambda_{1} \lambda_{2}} \frac{\lambda_{1}^{3} e^{\lambda_{2} x}-\lambda_{2}^{3} e^{\lambda_{1} x}}{\lambda_{1}^{2}-\lambda_{2}^{2}} .
$$

Substituting for $\lambda_{1}$ and $\lambda_{2}$, we obtain

$$
Y(x, s)=\Theta(s) V(x, s),
$$

where

$$
V(x, s)=(B / s)^{1 / 4} \exp \left(-X s^{1 / 4}\right) \sin \left(X s^{1 / 4}-\frac{1}{4} \pi\right)
$$

and $X=x(4 B)^{-1 / 4}$. In the special case that $\theta(t)=\theta_{\text {eq }}$ (so that $\Theta=\theta_{\text {eq }} / s$ ), the formula for $Y$ reduces to [6, Equation (29)]. Note also that we have mass conservation for any $\theta(t): \int_{0}^{\infty} Y(x, s) d x=0$. In particular, when $x=0$, (36) and (37) give

$$
Y(0, s)=-[B /(4 s)]^{1 / 4} \Theta(s) .
$$

In order to invert for $y(x, t)$, we consider a complex $s=S e^{i \sigma}$ with $S \geq 0$ and $-\pi<\sigma<\pi$. Then, our formulas for $Y$ and $V$ are valid in the whole complex $s$-plane, cut along the negative real $s$-axis. It is easier to work with $V$ rather than $Y$ (even for constant $\theta$ ) because $V$ is less singular at the branch point, $s=0$. The Bromwich inversion contour can then be deformed around the cut. Standard calculations give

$$
v(x, t)=-\frac{2 B}{\pi} \int_{0}^{\infty} k^{2} \cos k x e^{-k^{4} B t} d k,
$$

where the substitution $S=k^{4} B$ was used. Hence, the convolution theorem gives

$$
y(x, t)=\int_{0}^{t} \theta(t-\tau) v(x, \tau) d \tau,
$$

in agreement with (18). When $\theta(t)=\theta_{\text {eq }}$, we can integrate with respect to $\tau$; the result is (11). 
4.2. A generalized periodic problem. Let us generalize the periodic problem of Sec. 3 so that the slopes at $x= \pm \ell$ vary with time. Thus, we replace (20) and (21) by

$$
\partial y / \partial x=\mp \theta(t) \quad \text { at } x= \pm \ell, t>0 .
$$

Taking the Laplace transform with respect to $t$, we obtain (32); we seek a solution that is an even function of $x$ and that satisfies

$$
\partial Y / \partial x=-\Theta(s) \text { and } \partial^{3} Y / \partial x^{3}=0 \quad \text { at } x=\ell .
$$

The substitution $Y=\cos \lambda x$ in (32) leads to (34) with solutions $\lambda=\Lambda e^{i \varphi}$ given by (35). We pick two values of $\varphi, \varphi_{1}$ and $\varphi_{2}$, that give two different values of $\lambda, \lambda_{1}$ and $\lambda_{2}$, respectively, with $\lambda_{1} \neq-\lambda_{2}$ (because $\cos \lambda x$ is even in $\lambda$ ). We take $\varphi_{1}=\pi / 4$ and $\varphi_{2}=-\pi / 4$, giving $\lambda_{1}=(1+i) \Lambda / \sqrt{2}$ and $\lambda_{2}=\overline{\lambda_{1}}$. Thus, we have

$$
Y(x, s)=\mathcal{A}(s) \cos \lambda_{1} x+\mathcal{B}(s) \cos \lambda_{2} x,
$$

where $\mathcal{A}$ and $\mathcal{B}$ are determined using the boundary conditions (39), giving

$$
Y(x, s)=\frac{\Theta(s)}{\lambda_{1}^{2}-\lambda_{2}^{2}}\left\{\frac{\lambda_{1}^{2} \cos \lambda_{2} x}{\lambda_{2} \sin \lambda_{2} \ell}-\frac{\lambda_{2}^{2} \cos \lambda_{1} x}{\lambda_{1} \sin \lambda_{1} \ell}\right\} .
$$

Using the definitions of $\lambda_{1}$ and $\lambda_{2}$, we obtain (36) with

$$
V(x, s)=\frac{\cos \lambda_{1} x}{2 \lambda_{1} \sin \lambda_{1} \ell}+\frac{\cos \lambda_{2} x}{2 \lambda_{2} \sin \lambda_{2} \ell} .
$$

This function is analytic in the complex $s$-plane apart from simple poles at $s=-B(n \pi / \ell)^{4}$, $n=1,2, \ldots$. Again, we can check that mass is conserved for any $\theta(t): \int_{-\ell}^{\ell} Y(x, s) d x=0$.

Later, we shall require the solution at $x=\ell$; it is given by

$$
Y(\ell, s)=\Theta(s) \frac{\ell}{\Omega} \frac{\sin \Omega-\sinh \Omega}{\cosh \Omega-\cos \Omega} \quad \text { with } \Omega=\ell(4 s / B)^{1 / 4} .
$$

4.3. Two grooves. We consider two grooves, one at $x=\ell$ and one at $x=-\ell$. We are going to patch together two semi-infinite solutions (Mullins problem) with one cell of the periodic solution. If we did this using constant slopes at the interfaces, we would find that $y(x, t)$ would be discontinuous at the interfaces. The problem is symmetric about $x=0$, so consider the interface at $x=\ell$. On the right-hand side of this interface, we have

$$
\partial y / \partial x=\theta_{R}(t) \quad \text { at } x=\ell \text { for } t>0,
$$

and just to the left, we have

$$
\partial y / \partial x=-\theta_{L}(t) \quad \text { at } x=\ell \text { for } t>0,
$$

where $\theta_{R}$ and $\theta_{L}$ are unknown functions of $t$. If they were known, we could calculate $y(x, t)$ for $x>\ell$ using (a shifted version of) the generalized Mullins solution (Sec. 4.1), and we could calculate $y(x, t)$ for $|x|<\ell$ using the generalized periodic solution (Sec.4.2).

Now, two conditions have to be satisfied. First, $y$ must be continuous across $x=\ell$, so that

$$
y(\ell-, t)=y(\ell+, t) .
$$

Second, there is the thermodynamic condition

$$
\theta_{R}(t)+\theta_{L}(t)=\gamma_{i} / \gamma_{s} \equiv 2 \theta_{\mathrm{eq}}
$$


this reduces to (7) when $\theta_{R}=\theta_{L}$. These two conditions are easy to impose in the Laplace-transform domain. Thus, using (38) and (40), (41) gives

$$
\Theta_{R}(s)=\frac{\sinh \Omega-\sin \Omega}{\cosh \Omega-\cos \Omega} \Theta_{L}(s),
$$

whereas (42) immediately gives $\Theta_{R}(s)+\Theta_{L}(s)=2 \theta_{\text {eq }} / s$. These two equations can now be solved for $\Theta_{R}$ and $\Theta_{L}$. We choose to focus on $\Theta_{R}$, given by

$$
\Theta_{R}(s)=\frac{2 \theta_{\mathrm{eq}}}{s} \frac{\sinh \Omega-\sin \Omega}{F(\Omega)} \text { with } F(\Omega)=e^{\Omega}-\cos \Omega-\sin \Omega,
$$

where $\Omega=\ell(4 s / B)^{1 / 4}$. Once $\Theta_{R}(s)$ has been inverted for $\theta_{R}(t), \theta_{L}(t)$ is given by (42).

From (43), we find that $F$ has the expansion

$$
F(\Omega)=2 \Omega^{2} \sum_{n=0}^{\infty}\left(\frac{\Omega^{4 n}}{(4 n+2) !}+\frac{\Omega^{4 n+1}}{(4 n+3) !}\right)=\Omega^{2}\left(\Sigma_{0}+\Omega \Sigma_{1}\right),
$$

say, where

$$
\Sigma_{j}(s)=2 \sum_{n=0}^{\infty} \frac{\Omega^{4 n}}{(4 n+2+j) !}=2 \sum_{n=0}^{\infty} \frac{(4 / B)^{n} \ell^{4 n}}{(4 n+2+j) !} s^{n}, \quad j=0,1 .
$$

Notice that $\Sigma_{0}$ and $\Sigma_{1}$ are even functions of $\Omega$ and analytic functions of $s$. Explicitly, we have $\Sigma_{0}=(\cosh \Omega-\cos \Omega) / \Omega^{2}$ and $\Sigma_{1}=(\sinh \Omega-\sin \Omega) / \Omega^{3}$. Hence

$$
\Theta_{R}(s)=\frac{2 \theta_{\mathrm{eq}}}{s} \frac{\Omega \Sigma_{1}}{\Sigma_{0}+\Omega \Sigma_{1}} .
$$

This function is analytic in the whole complex $s$-plane, cut along the negative real $s$-axis. Deforming the Bromwich contour around the cut gives

$$
\theta_{R}(t)=\frac{\theta_{\mathrm{eq}}}{\pi} \int_{0}^{\infty} G(S) e^{-S t} d S
$$

where

$$
\begin{aligned}
G(S) & =-\frac{i}{S}\left\{\frac{(1+i) S^{1 / 4} \Lambda_{1}}{\Lambda_{0}+(1+i) S^{1 / 4} \Lambda_{1}}-\frac{(1-i) S^{1 / 4} \Lambda_{1}}{\Lambda_{0}+(1-i) S^{1 / 4} \Lambda_{1}}\right\} \\
& =\frac{2}{S} \frac{\Lambda_{0} \Lambda_{1} S^{1 / 4}}{\Lambda_{0}^{2}+2 \Lambda_{0} \Lambda_{1} S^{1 / 4}+2 \Lambda_{1}^{2} S^{1 / 2}},
\end{aligned}
$$

$\Lambda_{0}(S)=\Sigma_{0}(-S)$ and $\Lambda_{1}(S)=\ell B^{-1 / 4} \Sigma_{1}(-S)$.

Let us examine the behaviour of $\theta_{R}(t)$ for large times. For small $S$, the expansions (44) give $\Lambda_{0} \sim 1, \Lambda_{1} \sim \frac{1}{3} \ell B^{-1 / 4}$, and

$$
G(S) \sim \frac{2}{3} \ell B^{-1 / 4} S^{-3 / 4} \quad \text { as } S \rightarrow 0 .
$$

Then, Watson's lemma applied to (45) gives

$$
\theta_{R}(t) \sim \frac{2}{3}\left(\theta_{\text {eq }} / \pi\right) \Gamma\left(\frac{1}{4}\right) \ell(B t)^{-1 / 4} \quad \text { as } t \rightarrow \infty .
$$

Thus,

$$
\theta_{R} \rightarrow 0 \quad \text { and } \quad \theta_{L} \rightarrow 2 \theta_{\mathrm{eq}} \quad \text { as } t \rightarrow \infty .
$$

As expected, the groove root tilts until the semi-infinite surface profiles eliminate their curvature altogether. 
Let us study the time dependence of the groove root. At the interfaces, the groove depth can be calculated using either the generalized Mullins solution or the generalized periodic solution, because of the enforced continuity, (41). From (38), we obtain

$$
Y(\ell, s)=-\frac{\ell}{\Omega} \Theta_{R}(s)=-\frac{2 \theta_{\mathrm{eq}} \ell}{3 s}+\frac{2 \theta_{\mathrm{eq}} \ell}{3 s} \frac{\Sigma_{0}-3 \Sigma_{1}+\Omega \Sigma_{1}}{\Sigma_{0}+\Omega \Sigma_{1}} .
$$

The second term on the right-hand side can be treated as before; it leads to a contribution to $y(\ell, t)$ that decays as $(B t)^{-1 / 4}$ as $t \rightarrow \infty$. Hence

$$
y(\ell, t)=-\frac{2}{3} \theta_{\mathrm{eq}} \ell+\frac{2}{9}\left(\theta_{\mathrm{eq}} / \pi\right) \Gamma\left(\frac{1}{4}\right) \ell^{2}(B t)^{-1 / 4}+O\left(t^{-1 / 2}\right) \quad \text { as } t \rightarrow \infty .
$$

5. Conclusions. We have extended Mullins' linear theory of thermal surface grooving to periodic systems of grooves and to a pair of grooves. For periodic systems, we found the limiting profile and we showed that the Mullins solution gives a remarkably good approximation.

The two-groove system introduces asymmetry. We resolve potential discontinuities at the interfaces by allowing the groove-root systems to tilt by a time-dependent amount that we calculate. Note that, in reality, the groove roots may also move relative to each other; further investigation may reveal whether this possibility is important or not.

Our methods should also be useful for analysing surface grooving at interfaces between different materials. Again, the grooving will be asymmetric. For one study (both theoretical and experimental) of such a problem, see [7].

Acknowledgement. The problems discussed herein were brought to my attention by Moneesh Upmanyu (Division of Engineering, Colorado School of Mines, Golden, Colorado).

Appendix: Proof of Theorem 1, Take the Mellin transform of (30), defined by

$$
\widetilde{f}(z)=\int_{0}^{\infty} f(\xi) \xi^{z-1} d \xi
$$

$\widetilde{f}(z)$ is an analytic function of the complex variable $z$ for $\operatorname{Re}(z)>0$. Explicitly, we find that

$$
\widetilde{f}(z)=\sum_{n=1}^{\infty} \frac{1}{n^{2}} \int_{0}^{\infty} \xi^{z-1} e^{-\xi n^{4}} d \xi=\Gamma(z) \zeta(4 z+2),
$$

where $\Gamma(z)$ is defined by (13) and $\zeta(z)$ is the Riemann zeta function, defined by

$$
\zeta(z)=\sum_{n=1}^{\infty} \frac{1}{n^{z}} \quad \text { for } \operatorname{Re}(z)>1 .
$$

Equation (A.1) gives the analytic continuation of $\widetilde{f}(z)$ into $\operatorname{Re}(z) \leq 0$. The inversion formula gives

$$
f(\xi)=\frac{1}{2 \pi i} \int_{c-i \infty}^{c+i \infty} \widetilde{f}(z) \xi^{-z} d z \quad \text { with } c>0 .
$$

Moving the inversion contour to the left, we pick up residue contributions at the poles of $\widetilde{f}(z)$. It is known that $\Gamma(z)$ has simple poles at $z=-N, N=0,1,2, \ldots$ It is also 
known that $\zeta(z)$ has a simple pole at $z=1$ and satisfies $\zeta(-2 n)=0, n=1,2, \ldots$. Hence, $\widetilde{f}(z)$ has just two simple poles, one at $z=0$ with residue $\zeta(2)=\pi^{2} / 6$, and one at $z=-\frac{1}{4}$ with residue $\frac{1}{4} \Gamma\left(-\frac{1}{4}\right)=-\Gamma\left(\frac{3}{4}\right)$. The result (31) follows. Further details of the method used here can be found in $[5$.

\section{REFERENCES}

[1] P. Broadbridge and P. Tritscher, An integrable fourth-order nonlinear evolution equation applied to thermal grooving of metal surfaces, IMA J. Appl. Math. 53, 249-265 (1994) MR1314257(95j:35217)

[2] J. W. Cahn and J. E. Taylor, Surface motion by surface diffusion, Acta Metall. Mater. 42, 1045-1063 (1994)

[3] E. Fried and M. E. Gurtin, A unified treatment of evolving interfaces accounting for small deformations and atomic transport with emphasis on grain-boundaries and epitaxy, Adv. Appl. Mech. 40, $1-177(2004)$

[4] M. E. Gurtin, Thermomechanics of Evolving Phase Boundaries in the Plane, Oxford University Press, 1993 MR.1402243 (97k:73001)

[5] P. A. Martin, Asymptotic approximations for functions defined by series, with some applications to the theory of guided waves, IMA J. Appl. Math. 54, 139-157 (1995) MR 1334457(96f:41024)

[6] W. W. Mullins, Theory of thermal grooving, J. Appl. Phys. 28, 333-339 (1957)

[7] E. Rabkin, L. Klinger, T. Izyumova, A. Berner, and V. Semonov, Grain boundary grooving with simultaneous grain boundary sliding in Ni-rich NiAl, Acta Mater. 49, 1429-1438 (2001)

[8] W. M. Robertson, Grain-boundary grooving by surface diffusion for finite surface slopes, J. Appl. Phys. 42, 463-467 (1971)

[9] P. Tritscher and P. Broadbridge, Grain boundary grooving by surface diffusion: an analytic nonlinear model for a symmetric groove, Proc. Roy. Soc. A 450, 569-587 (1995) 